\title{
Profit Analysis of a Two-Unit Cold Standby System with Delayed Repair of Partially Failed Unit and Better Utilization of Units
}

\author{
Praween Kumar \\ Department of Mathematics \\ Bharat Institute of Technology \\ Meerut, UP, India
}

\author{
Anu Sirohi \\ Department of Mathematics \\ Bharat Institute of Technology \\ Meerut, UP, India
}

\begin{abstract}
The paper presents the profit analysis of a two unit cold standby system with delayed repair of partially failed unit and better utilization of units. It is assumed that the system stops functioning only when both the units fail. The failure time distributions and delayed time distribution of each unit are assumed to be negative exponential with different parameters whereas the repair time distributions are taken to general. Formulas for various reliability indices of the system including availability, busy period analysis of the repairman, and profit function analysis by using the regenerative point technique are derived.
\end{abstract}

\section{General Terms}

Redundancy; Standby system; Stochastic; Failure and Repair times; Correlation.

\section{INTRODUCTION}

No doubt that the two-unit cold standby systems have attracted the attention of several researchers including [1-3, 5, $7,11]$ working in the field of reliability theory. They have considered the concepts like random inspection of the units, random shocks, allowed down time, imperfect and slow switching device, random check of standby units etc. Some authors including Gupta et. al. [3-7, 10] analyzed two-unit cold standby system models assuming the three modes of each unit-Normal (N), Partial failure (P) and Total failure (F).They have considered a single repairman who repairs a unit failed partially or totally under the stringent assumptions

(i) The repair of a partially failed unit may be performed during its operation.

(ii) The partially failed unit operates to run the system while the other (standby) unit is available in its normal mode.

Many practical situations can be visualized when above assumptions can not be imposed on the systems i.e. repair and operation of a partially failed unit are not possible simultaneously and to get the full efficiency of the system, the unit available in normal mode operates rather than the unit in partial failure mode.

One way to improve the system reliability is to add redundant components. Standby redundancy is a technique widely used to improve system reliability and availability [8-9, 12]. In general there are three types in redundancy i.e. cold, hot, and warm standby. Cold standby implies that the inactive components have a zero failure rate and cannot fail while in standby state. Hot standby implies that an inactive component has the same failure rate as when it is in operation. Warm standby is an intermediate case and it implies that an inactive component has a failure rate between that for the cold and hot standby. It is also called dormant failure in some papers.
Keeping the above facts in view, we in the present paper analyze a two-unit cold standby system model relaxing the above assumptions. In this, it is supposed that the repairman is not always with the system when a unit fails partially. But when a unit fails totally, he becomes available instantaneously to repair the totally failed unit and then he remains with the system till all the partially or totally failed units are repaired.

\section{MODEL DESCRIPTION}

\subsection{System Description}

There are some real systems such as power generator and transmission systems where some new equipment groups need to be added because of the requirement for more output of the system. In this model, initially the system comprises two identical units. The satisfactory operation of any of the unit is sufficient to do the job. Each unit of the system has three modes-normal, partial failure and total failure (N, P and F). The failure time distributions and delayed time distribution of each unit are assumed to be negative exponential distribution with different parameters whereas the repair time distributions are taken generals.

\subsection{Assumptions}

1. It is a general cold standby system comprises two identical units, each having three modes: Normal $(\mathrm{N})$, partial failure $(\mathrm{P})$, and total failure $(\mathrm{F})$. Initially one unit is operative and the other is kept into cold standby.

2. Each unit first enters into P-mode and then into F-mode i.e. a unit can't enter into F-mode without passing through P-mode.

3. Repairman is not always with the system, but a single repairman can be made available whenever required.

4. When a unit fails partially, repairman is intimated and he takes a significant time to be available with the system and this time is named as delayed time. However, when a unit fails totally, the repairman visits instantaneously.

5. The priority in operation is being given to $\mathrm{N}$-unit over the P-unit. A P-unit operates only when the other unit is either in P-mode or in F-mode.

6. The switching device is always perfect and instantaneous.

7. The online repair of a $\mathrm{P}$-unit is not possible.

8. If a P-unit is under repair while the other unit enters into the F-mode from P-mode, the repair of the P-unit is stopped and it starts operating while the other unit in Fmode undergoes repair. The discontinued repair of the $\mathrm{p}$ unit is of preemptive repeat type. 


\section{NOTATIONS AND STATES OF THE SYSTEM}

To write the states of the system we define following symbols for both the units:

$* /$ transform of a function.

: Symbol for Laplace/ Laplace Stieltjes

$\mathrm{N}_{\mathrm{o}}, \mathrm{N}_{\mathrm{s}}$ : Unit in N-mode and operative, standby.

$\mathrm{P}_{\mathrm{o}}, \mathrm{P}_{\mathrm{r}} \quad$ : Unit in P-mode and operative, under repair.

$\mathrm{P}_{\mathrm{dr}} \quad$ : Unit in P-mode and under delayed in repair.

$\mathrm{F}_{\mathrm{r}}, \mathrm{F}_{\mathrm{w}} \quad$ : Unit in F-mode and under repair, waiting for repair.

$\alpha_{1}, \alpha_{2}$ : Constant failure rates of a unit from $\mathrm{N}$-mode to $\mathrm{P}$ mode, P-mode to F-mode.

$\theta \quad:$ Parameter of the negative exponential delayed time distribution.

$\mathrm{g} 1$ (.), G1 (.) : p.d.f and c.d.f of repair time distribution of a Punit.

g2 (.), G2 (.) : p.d.f and c.d.f of repair time distribution of a Funit.

Considering the above symbols in view of the assumptions we have the following states of the system-

Up States (with full efficiency)

$\mathrm{SO}=(\mathrm{No}, \mathrm{Ns})$

$\mathrm{S} 1=(\mathrm{Pdr}, \mathrm{No})$

$\mathrm{S} 3=(\mathrm{Pr}, \mathrm{No})$

$\mathrm{S} 7=(\mathrm{Fr}, \mathrm{No})$

Up States (with reduced efficiency)

$\mathrm{S} 2=(\mathrm{Pdr}, \mathrm{Po})$

$\mathrm{S} 4=(\mathrm{Pr}, \mathrm{Po})$

$\mathrm{S} 5=(\mathrm{Po}, \mathrm{Fr})$

\section{Failed States}

$\mathrm{S} 6=(\mathrm{Fw}, \mathrm{Fr})$

The transition diagram of the system model along with failure time variables, and repair rates is shown in fig 1. In figure we observe that the epochs of transition from S3 to S4, S5 to S6, and S7 to S5 are non-regenerative points. All the other epochs are regenerative.

\section{TRANSITION PROBABILITIES AND SOJOURN TIMES}

Let $Q_{i j}(t)$ is the one step unconditional transition probability, which can be obtained by using the simple probabilistic arguments, as follows-

$$
\begin{aligned}
Q_{01}(t) & =\int_{0}^{t} P\left[\begin{array}{l}
\text { system transits from } \mathrm{S}_{0} \text { to } \mathrm{S}_{1} \\
\text { during } \mathrm{u}, \mathrm{u}+\mathrm{du}
\end{array}\right] \\
& =\int_{o}^{t} \alpha_{1} e^{-\alpha_{1} u} d u=1-e^{-\alpha_{1} t}
\end{aligned}
$$

Similarly other probabilistic arguments can be obtained, then the non-zero elements of the transition probability matrix $\mathrm{P}=$ $\left(\mathrm{p}_{\mathrm{ij}}\right)$ for the model are as follows-

$$
\begin{aligned}
& p_{01}=1, p_{12}=\frac{\alpha_{1}}{\alpha_{1}+\theta}, p_{13}=\frac{\theta}{\alpha_{1}+\theta} \\
& p_{24}=\frac{\theta}{\alpha_{2}+\theta}, \quad p_{25}=\frac{\alpha_{2}}{\alpha_{2}+\theta}, p_{30}=\tilde{G}_{1}\left(\alpha_{1}\right) \\
& p_{33}^{(4)}=\frac{\alpha_{1}\left[G_{1}^{\circ}\left(\alpha_{2}\right)-G_{1}^{\circ}\left(\alpha_{1}\right)\right]}{\alpha_{1}-\alpha_{2}} \\
& p_{35}^{(4)}=\frac{\alpha_{1} \alpha_{2}}{\alpha_{1}-\alpha_{2}}\left[\frac{1-G_{1}^{\circ}\left(\alpha_{2}\right)}{\alpha_{2}}-\frac{1-G_{1}^{\circ}\left(\alpha_{2}\right)}{\alpha_{1}}\right] \\
& p_{43}=\tilde{G}_{1}\left(\alpha_{2}\right), p_{45}=1-\tilde{G}_{1}\left(\alpha_{2}\right), p_{53}=\tilde{G}_{2}\left(\alpha_{2}\right) \\
& p_{56}=p_{57}^{(6)}=1-G_{2}^{\circ}\left(\alpha_{2}\right), p_{70}=\tilde{G}_{2}\left(\alpha_{1}\right), \\
& p_{73}^{(5)}=\frac{\alpha_{1}}{\alpha_{1}-\alpha_{2}}\left[G_{2}^{\circ}\left(\alpha_{2}\right)-G_{2}^{\circ}\left(\alpha_{1}\right)\right. \\
& p_{77}^{(5,6)}=1-\frac{\alpha_{1}}{\alpha_{1}-\alpha_{2}} G_{2}^{o}\left(\alpha_{2}\right)+\frac{\alpha_{2}}{\alpha_{1}-\alpha_{2}} G_{2}^{o}\left(\alpha_{1}\right)
\end{aligned}
$$

We observe the following relations-

$$
\begin{aligned}
& p_{12}+p_{13}=1=p_{01}=p_{67} \\
& p_{24}+p_{25}=1=p_{30}+p_{33}^{(4)}+p_{35}^{(4)} \\
& p_{43}+p_{45}=1=p_{53}+p_{57}^{(6)}\left(=p_{56}\right)=1 \\
& p_{70}+p_{73}^{(5)}+p_{77}^{(5,6)}=p_{70}+p_{75}
\end{aligned}
$$

Let $\mathrm{X}_{\mathrm{i}}$ denotes the sojourn time in state $\mathrm{S}_{\mathrm{i}}$, then the mean sojourn time in state $\mathrm{S}$ is given by-

$$
\psi_{i}=\int P\left(X_{i} \phi t\right) d t
$$

The mean sojourn times in various states are as follows:

$$
\begin{aligned}
& \psi_{0}=\frac{1}{\alpha_{1}}, \psi_{1}=\frac{1}{\alpha_{1}+\theta}, \psi_{2}=\frac{1}{\alpha_{2}+\theta} \\
& \psi_{3}=\int e^{-\alpha_{1} t} \bar{G}_{1}(t) d t, \quad \psi_{4}=\int e^{-\alpha_{2} t} \bar{G}_{1}(t) d t \\
& \psi_{5}=\int e^{-\alpha_{2} t} \bar{G}_{2}(t) d t, \quad \psi_{6}=\int \bar{G}_{2}(t) d t \\
& \psi_{7}=\int e^{-\alpha_{1} t} \bar{G}_{2}(t) d t
\end{aligned}
$$

The unconditional mean time taken by the system to transit from any regenerative. State $\mathrm{Si}$ when time is counted from epoch of entrance into state $\mathrm{Sj}$ is given by

$$
m_{i j}=\int t d Q_{i j}(t)=-\left[\frac{d}{d s}\left(Q_{i j}^{* *}(s)\right)\right]_{s=0}
$$

We have

$$
\begin{aligned}
& m_{01}=\psi_{0}, \quad m_{12}+m_{13}=\psi_{1}, m_{24}+m_{25}=\psi_{2} \\
& m_{30}+m_{33}^{(4)}+m_{35}^{(4)}=\frac{\alpha_{1}}{\alpha_{1}-\alpha_{2}} \psi_{4}-\frac{\alpha_{2}}{\alpha_{1}-\alpha_{2}} \psi_{3}=n_{1} \text { (say) }
\end{aligned}
$$


$m_{43}+m_{45}=\psi_{4}, m_{53}+m_{57}^{(6)}=\int t d G_{2}(t)=n_{2}$

$m_{70}+m_{73}^{(5)}+m_{77}^{(5,6)}=\int t d G_{2}(t)=n_{2}$

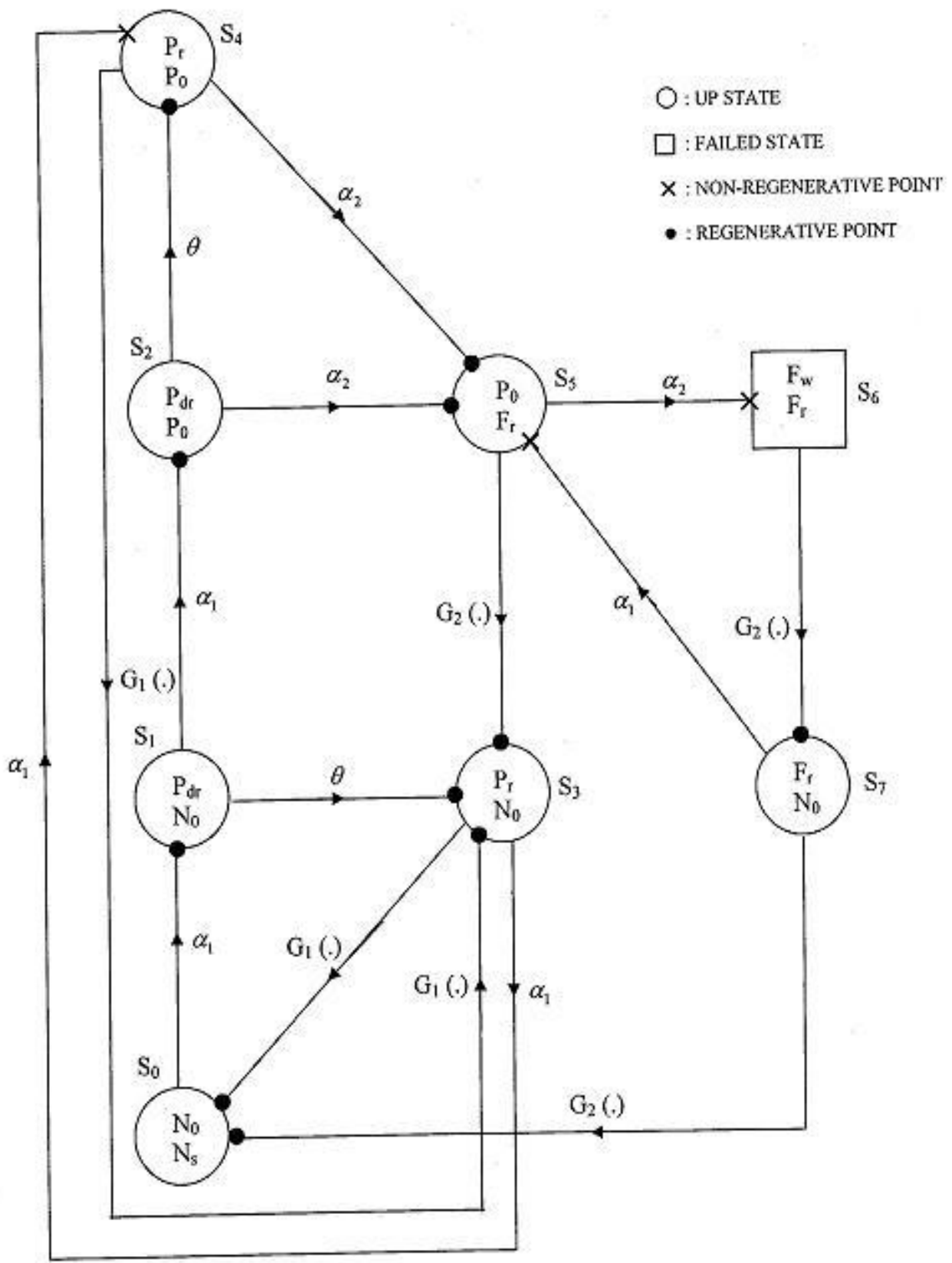

Fig 1: State Transition Diagram

\section{RELIABILITY AND MTSF}

Let the r.v $\mathrm{T}_{\mathrm{i}}$ be the time to system failure when the system starts its operation from state $S_{i} \in E$, then the reliability of the system is given by-

$R_{i}(t)=P\left(T_{i} \phi t\right)$

To given $R_{i}(t)$, we assume that failed state $S_{6}$ as absorbing of the system. Using the simple probabilistic arguments, one can easily develop the recurrence relations among $R_{i}(t) ; i=0,1,2$, 3,4 , 5. Taking the Laplace transformation of the relations and simplifying the resulting set of algebraic equations for $R_{0}^{*}(s)$, we get after omitting the argument 's' for brevity-

$$
R_{0}^{*}(s)=\frac{N_{1}(s)}{D_{1}(s)}
$$


Where,

$$
\begin{aligned}
& N_{1}(s)=\left(Z_{0}^{*}+q_{01}^{*} Z_{1}^{*}+q_{01}^{*} q_{12}^{*} Z_{2}^{*}\right) \\
& \left(1-q_{33}^{(4) *}-q_{35}^{(4)} q_{53}^{*}\right)+q_{01}^{*}\left(Z_{3}^{*}+q_{34}{ }^{*} Z_{4}^{*}\right) \\
& {\left[q_{12}^{*}\left\{q_{24}^{*}\left(q_{43}^{*}+q_{45}^{*} q_{53}^{*}\right)+q_{25}^{*} q_{53}^{*}\right\}+q_{13}^{*}\right]} \\
& +q_{01}^{*} q_{12}^{*} q_{24}^{*} Z_{4}^{*}\left(1-q_{33}^{(4)^{*}}-q_{35}^{(4)^{*}} q_{53}^{*}\right)+ \\
& q_{01}^{*} Z_{5}^{*}\left\{\begin{array}{l}
q_{12}^{*} q_{24}^{*}\left(q_{45}^{*}-q_{33}^{(4)^{*}} q_{45}^{*}+q_{43}^{*} q_{35}^{(4)^{*}}\right) \\
+q_{12}^{*} q_{25}^{*}\left(1-q_{33}^{(4)^{*}}\right)+q_{13}^{*} q_{35}^{(4)^{*}}
\end{array}\right\} \\
& D_{1}(s)=\left(1-q_{33}^{(4)^{*}}-q_{35}^{(4)^{*}} q_{53}^{*}\right)-q_{30}^{*} q_{01}^{*} \\
& {\left[q_{12}^{*}\left\{q_{24}^{*}\left(q_{43}^{*}+q_{45}^{*} q_{53}^{*}\right)+q_{25}^{*} q_{53}^{*}\right\}+q_{13}^{*}\right]}
\end{aligned}
$$

Taking inverse Laplace transformation of (4), we get the reliability of the system. The Mean Time to System Failure (MTSF) is given by-

$$
\begin{aligned}
& E\left(T_{0}\right)=\int R_{0}(t) d t=\left.R_{0}^{*}(s)\right|_{s=0}=\frac{N_{1}(0)}{D_{1}(0)} \\
& N_{1}(\mathrm{O})=\left(\psi_{0}+\psi_{1}+p_{12} \psi_{2}\right) \\
& \left(1-p_{33}^{(4)}-p_{35}^{(4)} p_{53}\right)+\left(\psi_{3}+p_{34} \psi_{4}\right) \\
& \text { * }\left[p_{12}\left\{p_{24}\left(p_{43}+p_{45} p_{53}\right\}+p_{53}\right]\right. \\
& +p_{12} p_{24} \psi_{4}\left(1-p_{33}^{(4)}-p_{35}^{(4)} p_{53}\right) \\
& +\psi_{5}\left[\begin{array}{l}
p_{12} p_{24}\left(p_{45}-p_{45} p_{33}^{(4)}+p_{43} p_{35}^{4}\right) \\
+p_{12} p_{25}\left(1-p_{33}^{(4)}+p_{13} p_{35}^{(4)}\right)
\end{array}\right] \\
& \begin{array}{l}
D_{1}(0)=\left(1-p_{33}^{(4)}-p_{35}^{(4)} p_{53}\right)-p_{30} \\
\text { And } \\
{\left[p_{12}\left\{p_{24}\left(p_{43}+p_{45} p_{53}\right)+p_{25} p_{53}\right\}+p_{13}\right]}
\end{array}
\end{aligned}
$$

\section{Steady State Availability}

Let $A_{i}^{f}(t)$ and $A_{i}^{r}(t)$ be the probability that the system is up at epoch $t$ with full efficiency and reduced efficiency respectively when initially it starts functioning from state $S_{i} \in E$ by using probabilistic arguments we get

$$
A_{i}^{f}(t)-\quad A_{0}^{f \bullet}(s)=\frac{N_{2}(s)}{D_{2}(s)}
$$

Where,

$$
\begin{aligned}
& N_{2}(s)=\left(Z_{0}^{*}+q_{01}^{*} Z_{1}^{*}\right) \\
& {\left[\left(1-q_{77}^{(5,6)}\right)\left(1-q_{33}^{(4)}-q_{53} q_{35}^{(4)}\right)-q_{73}^{(5)} q_{35}^{(4)} q_{57}^{(6)}\right]} \\
& +Z_{3}^{*} q_{01}^{*}\left[q _ { 1 2 } ^ { * } q _ { 2 4 } ^ { * } \left\{q_{43}^{*}\left(1-q_{77}^{(5,6)^{*}}\right)\right.\right. \\
& \left.+q_{45}^{*} q_{53}^{*}\left(1-q_{77}^{(5,6)^{*}}\right)+q_{45}^{*} q_{57}^{(6)} q_{73}^{(5)^{*}}\right\} \\
& +q_{12}^{*} q_{25}^{*}\left\{q_{53}^{*}\left(1-q_{77}^{(5,6)^{*}}\right)+q_{57}^{6^{*}} q_{73}^{(5)^{*}}\right\} \\
& \left.+q_{13}^{*}\left(1-q_{77}^{(5,6)^{*}}\right)\right]+q_{01}^{*} q_{57}^{(6)^{*}} Z_{7}^{*} \\
& {\left[\begin{array}{l}
\left.q_{12}^{*}\left\{\left(1-q_{33}^{(4)^{*}}\right)\left(q_{24}^{*} q_{45}^{*}+q_{25}^{*}\right)+q_{24}^{*} q_{43}^{*} q_{35}^{(4)^{*}}\right\}\right] \\
+q_{13}^{*} q_{35}^{(4)^{*}}
\end{array}\right.}
\end{aligned}
$$

and

$$
\begin{aligned}
& D_{2}(s)=\left[\left(1-q_{77}^{(5,6)^{*}}\right)\left(1-q_{33}^{(4)^{*}}-q_{53}^{*} q_{35}^{*}\right)-q_{73}^{(5)^{*}} q_{35}^{(4)^{*}} q_{57}^{(6)^{*}}\right] \\
& -q_{30}^{*} q_{01}^{*}\left[q _ { 1 2 } ^ { * } q _ { 2 4 } ^ { * } \left\{q_{43}^{*}\left(1-q_{77}^{(5,6)^{*}}\right)+q_{45}^{*} q_{53}^{*}\left(1-q_{77}^{(5,6)^{*}}\right)\right.\right. \\
& \left.+q_{45}^{*} q_{57}^{(6)^{*}} q_{73}^{(5)^{*}}\right\}+q_{12}^{*} q_{25}^{*}\left\{q_{53}^{*}\left(1-q_{77}^{(5,6)^{*}}\right)+q_{57}^{(6)^{*}} q_{73}^{(5) *}\right\} \\
& \left.+q_{13}^{*}\left(1-q_{77}^{(5,6)^{*}}\right)\right]-q_{01}^{*} q_{57}^{(6)^{*}} q_{70}^{*} \\
& {\left[\begin{array}{l}
\left.q_{12}^{*}\left\{\left(1-q_{33}^{(4)^{*}}\right)\left(q_{24}^{*} q_{45}^{*}+q_{25}^{*}\right)+q_{24}^{*} q_{43}^{*} q_{35}^{(4)^{*}}\right\}\right] \\
+q_{13}^{*} q_{35}^{(4) *}
\end{array}\right]}
\end{aligned}
$$

Similarly, the point wise availabilities $A_{i}^{r}(t)$, i.e. when system is up with reduced efficiency (in partial failure mode) can also be obtained and are as follows-

$A_{0}^{r^{*}}(s)=\frac{N_{3}(s)}{D_{2}(s)}$ Where,

$N_{3}(s)=q_{01}^{*} q_{12}^{*}\left(Z_{2}^{*}+q_{24}^{*} Z_{4}^{*}\right)$

$\left[\left(1-q_{77}^{(5,6)^{*}}\right)\left(1-q_{33}^{(4)^{*}}-q_{53}^{*} q_{35}^{(4)^{*}}\right)-q_{73}^{(5)^{*}} q_{35}^{(4)^{*}} q_{57}^{(6)^{*}}\right]$

$+q_{01}^{*} q_{34}^{*} Z_{4}^{*}\left[q_{12}^{*} q_{24}^{*}\left\{\left(1-q_{77}^{(5,6)^{*}}\right)\left(q_{43}^{*}+q_{45}^{*} q_{53}^{*}\right)+q_{45}^{*} q_{57}^{(6)^{*}} q_{73}^{(5)^{*}}\right\}\right.$

$\left.+q_{12}^{*} q_{25}^{*}\left\{\left(1-q_{77}^{(5,6)^{*}}\right)\left(q_{53}^{*}+q_{57}^{(6)^{*}} q_{73}^{(5)^{*}}\right)+q_{13}^{*}\left(1-q_{77}^{(5,6)^{*}}\right)\right\}\right]$

$+q_{01}^{*}\left(1-q_{77}^{(5,6)^{*}}+q_{57}^{(6)^{*}} q_{75}^{*}\right) Z_{5}^{*}$

$\left[q_{12}^{*}\left\{\left(1-q_{33}^{(4)^{*}}\right)\left(q_{24}^{*} q_{45}^{*}+q_{25}^{*}\right)+q_{24}^{*} q_{43}^{*} q_{35}^{(4)^{*}}\right\}+q_{13}^{*} q_{35}^{(4)}\right]$

Now to obtain the steady-state probabilities that the system will be operative in normal and partial failure mode, we proceed as follows $\rightarrow$

$$
Z_{i}^{*}(0)=\int Z_{i}(t) d t=\psi_{i} \quad(i=0,1,2,3,4,5,7)
$$

Using the result $q_{i j}^{*}(0)=p_{i j}$, we have $D_{2}(0)=0$

Therefore, the steady state probability that the system will be operative in normal mode (with full efficiency) is given by-

$$
A_{0}^{f}=\lim (t \rightarrow \infty) \frac{N_{2}(t)}{D_{2}(t)}=\lim (s \rightarrow 0) s \frac{N_{2}(s)}{D_{2}(s)}=\frac{N_{2}(0)}{D_{2}^{\prime}(0)}
$$

By using L'hospital's rule. Where, 
$N_{2}(0)=\left(\psi_{0}+\psi_{1}\right)\left[\begin{array}{l}\left(1-p_{77}^{(5,6)}\right)\left(1-p_{33}^{(5,4)}-p_{53} p_{35}^{(4)}\right) \\ -p_{35}^{(4)} p_{57}^{(6)} p_{73}^{(5)}\end{array}\right]$

$+\psi_{3}\left[p_{12} p_{24}\left\{\left(1-p_{77}^{(5,6)}\right)\left(p_{43}+p_{45} p_{53}\right)+\right.\right.$

$\left.p_{45} p_{57}^{(6)} p_{73}^{(5)}\right\}+p_{12} p_{25}\left\{p_{53}\left(1-p_{77}^{(5,6)}\right)+p_{57}^{(6)} p_{73}^{(5)}\right\}+$

$\left.p_{13}\left(1-p_{77}^{(5,6)}\right)\right]+p_{57}^{(6)} \psi_{7}\left[p_{12}\left\{\left(1-p_{33}^{(4)}\right)\left(p_{24} p_{45}+p_{25}\right)+\right.\right.$ $\left.\left.p_{24} p_{43} p_{35}^{(4)}\right\}+p_{13} p_{35}^{(4)}\right]$

Similarly the steady state probability that the system will be operative in partial failure mode (with reduced efficiency) is given by

$A_{0}^{r}=\lim (t \rightarrow \infty) \frac{N_{3}(t)}{D_{2}(t)}=\lim (s \rightarrow 0) s \frac{N_{3}(s)}{D_{2}(s)}=\frac{N_{3}(0)}{D_{2}^{\prime}(0)}$

By using L'hospital's rule. Where,

$N_{3}(0)=\left(\psi_{0}+\psi_{1}\right)\left[\left(1-p_{77}^{(5,6)}\right)\left(1-p_{33}^{(5,4)}-p_{53} p_{35}^{(4)}\right)-p_{35}^{(4)} p_{57}^{(6)} p_{73}^{(5)}\right]$

$+\psi_{3}\left[p_{12} p_{24}\left\{\left(1-p_{77}^{(5,6)}\right)\left(p_{43}+p_{45} p_{53}\right)+\right.\right.$

$\left.p_{45} p_{57}^{(6)} p_{73}^{(5)}\right\}+p_{12} p_{25}\left\{p_{53}\left(1-p_{77}^{(5,6)}\right)+\right.$

$\left.\left.p_{57}^{(6)} p_{73}^{(5)}\right\}+p_{13}\left(1-p_{77}^{(5,6)}\right)\right]+p_{57}^{(6)} \psi_{7}\left[p_{12}\left\{\left(1-p_{33}^{(4)}\right)\right.\right.$

$\left.\left.\left(p_{24} p_{45}+p_{25}\right)+p_{24} p_{43} p_{35}^{(4)}\right\}+p_{13} p_{35}^{(4)}\right]$

\section{PROFIT FUNCTION ANALYSIS}

The net expected profit incurred in $(0, t)$ is given by

$P=K_{0} \mu_{u p}^{f}(t)+K_{1} \mu_{u p}^{r}(t)-K_{2} \mu_{b u s y}^{p}(t)-K_{3} \mu_{b u s y}^{f}(t)$

$-K_{4} V_{0}(t)$

where

$\mathrm{K}_{0}=$ per unit up time revenues by the system when it works with full efficiency.

$\mathrm{K}_{1}=$ per unit up time revenues by the system when it works with reduced efficiency.

$\mathrm{K}_{2}=$ amount spend per unit of time in repairing the P-unit.

$\mathrm{K}_{3}=$ amount spend per unit of time in repairing the F-unit.

$\mathrm{K} 4=$ payment to the repairman for his instantaneous visit at the system.

Similarly other related terms can be evaluated. Now the expected profit per unit time in steady state is given by

$$
\begin{aligned}
P & =\lim _{t \rightarrow \infty} \frac{P(t)}{t}=\lim _{s \rightarrow 0} s^{2} P^{*}(s) \\
& =K_{0} A_{0}^{f}+K_{1} A_{0}^{r}-K_{2} B_{0}^{p}-K_{3} B_{0}^{f}-K_{4} V_{0}
\end{aligned}
$$

\section{PARTICULAR CASE}

When the repair times of $\mathrm{P}$-unit and F-unit also follows the negative exponential distribution with parameters $\beta_{1}$ and $\beta_{2}$ respectively, i.e.

$G_{i}(t)=1-e^{-\beta_{i}(t)} ; \mathrm{i}=1,2$ So that $\tilde{G}_{i}(t)=\frac{\beta_{i}}{s+\beta_{i}}$
Then, we have the following changes-

$$
\begin{aligned}
& p_{30}=\frac{\beta_{1}}{\alpha_{1}+\beta_{1}}, p_{33}^{(4)}=\frac{\alpha_{1} \beta_{1}}{\left(\alpha_{1}+\beta_{1}\right) \cdot\left(\alpha_{2}+\beta_{1}\right)}, \\
& p_{35}^{(4)}=\frac{\alpha_{1} \alpha_{2}}{\left(\alpha_{1}+\beta_{1}\right)\left(\alpha_{2}+\beta_{1}\right)}, p_{43}=\frac{\beta_{1}}{\alpha_{2}+\beta_{1}} \\
& p_{45}=\frac{\alpha_{2}}{\alpha_{2}+\beta_{1}}, p_{53}=\frac{\beta_{2}}{\alpha_{2}+\beta_{2}} \\
& p_{56}=p_{57}^{(6)}=\frac{\alpha_{2}}{\alpha_{2}+\beta_{2}}, p_{70}=\frac{\beta_{2}}{\alpha_{1}+\beta_{2}} \\
& p_{73}^{(5)}=\frac{\alpha_{1} \beta_{2}}{\left(\alpha_{1}+\beta_{2}\right)\left(\alpha_{2}+\beta_{2}\right)}
\end{aligned}
$$

$$
\begin{aligned}
& p_{77}^{(5,6)}=\frac{\alpha_{1} \alpha_{2}}{\left(\alpha_{1}+\beta_{2}\right)\left(\alpha_{2}+\beta_{2}\right)}, p_{75}=\frac{\alpha_{1}}{\alpha_{1}+\beta_{2}} \\
& \psi_{3}=\frac{1}{\alpha_{1}+\beta_{1}}, \psi_{4}=\frac{1}{\alpha_{2}+\beta_{1}}, \psi_{5}=\frac{1}{\alpha_{2}+\beta_{2}}, \\
& \psi_{7}=\frac{1}{\alpha_{1}+\beta_{2}}
\end{aligned}
$$

\section{CONCLUSION}

To study the system behavior through graphs we plot the curves for MTSF and profit function in fig 2 and fig 3 respectively w.r.t $\alpha_{1}$ for three different values of repair rate $\beta_{1}$ and two different values of $\theta$ (rate of availability of repairman) whereas the other parameters are kept fixed as $\alpha_{2}=0.15, \beta_{2}=0.10, K_{0}=500, K_{1}=300, K_{2}=100$,

$K_{3}=150, K_{4}=75$. From fig 1.2 , it is evident that the MTSF decreases uniformly as $\alpha_{1}$ (failure rate) increases. It is also observed that the MTSF increases with the increases of rate of availability of repairman $(\theta)$ and with the increase of repair rate $\beta_{1}$. From fig1.3, it is obvious that the profit decreases linearly for $\beta=0.025$ as the failure rate $\alpha_{1}$ increases and the degree of this decrement increases as the values of $\beta_{1}$ increase from 0.025 . Further, we note that as the value of $\beta_{1}$ increases, the profit also increases and it decreases with the increase in $(\theta)$.

\section{ACKNOWLEDGMENTS}

Our thanks to the Prof. Rakesh Gupta, Professor, Institute of Advanced Studies, CCS University who was always helpful as and when required.

\section{REFERENCES}

[1] Singh, S.K., Rakesh Gupta., and Goel, L.R., (1985), "Cost analysis of a two-unit cold standby system with two types of operation and repair". Microelectron, Reliability, 25(1), 71-75. 
[2] Gupta, S.M., Jaiswal, N.K., and Goel, L.R., (1983), "Stochastic behavior of a two-unit cold standby system with three modes and allowed down time". Microelectron, Reliability, 23(2), 333-336.

[3] Gupta, R., and Bansal, S., (1990),"Cost benefit analysis of two-unit cold standby system with the provision of rest to a unit". International journal of system science, 21, 1451-1462.

[4] Goel, L.R., Tyagi, P.K., and Gupta, R., (1994),"Analysis of a standby system with dependent repair time and slow switching device" Microelectron, Reliability, 34(2), 383386.

[5] Singh, S.K., Rakesh Gupta., and Goel, L.R., (1983), "Analysis of a two-unit cold standby system with three modes". Microelectron, Reliability, 23, 1041-1044.

[6] Goel, L.R., and Gupta, P., (1984),"Switch failure in a two-unit standby system with better utilization of units". Microelectron, Reliability, 24, 439-443.

[7] Goel, L.R., Gupta, R., and Singh, S.K., (1985),'Profit analysis of a cold standby with two repair distributions". Microelectron, Reliability, 25, 467-472.

[8] Goel, L.R., and Srivastava, P., (1991),"Profit analysis of two unit redundant system with provision of rest and correlated failures and repairs". Microelectron, Reliability, 31, 827-833.

[9] Kumar, Praween and Sharma, S. K. (2006). A two nonidentical unit parallel system with inspection and correlated lifetimes. Journal of Rajasthan Academy of Physical Sciences, 5 (3), 301-318.

[10] Kumar, Praween and Gupta, R (2007). Reliability analysis of a single unit $\mathrm{M}|\mathrm{G}| 1$ system model with helping unit. Journal of Combinatorics, Information \& System Sciences, J. of Comb. Info. \& System Sciences, Vol. 32, No. 1-4, pp. 209-219.

[11] Kumar J., Kadyan M. S., Malik S. C., (2012), Cost Analysis of a Two-Unit Cold Standby System Subject to Degradation, Inspection and Priority, Eksploatacja Niezawodnosc - Maintenance and Reliability. 14(4), 278-283.

[12] Kumar, Praween and Sirohi, A., (2014) Stochastic Analysis of a Two Priority Unit Standby System with Imperfect Switch and Correlated Failure and Repair Times. Int. journal of Adv Research in com sc and software engineering, 4 (8), 621-628.

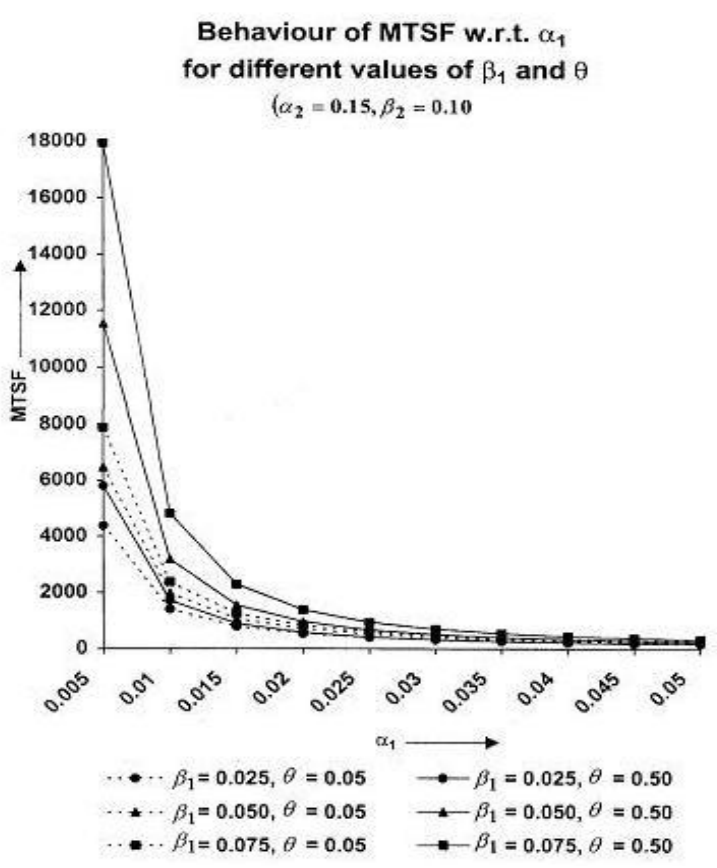

Fig 2: Behavior of MTSF

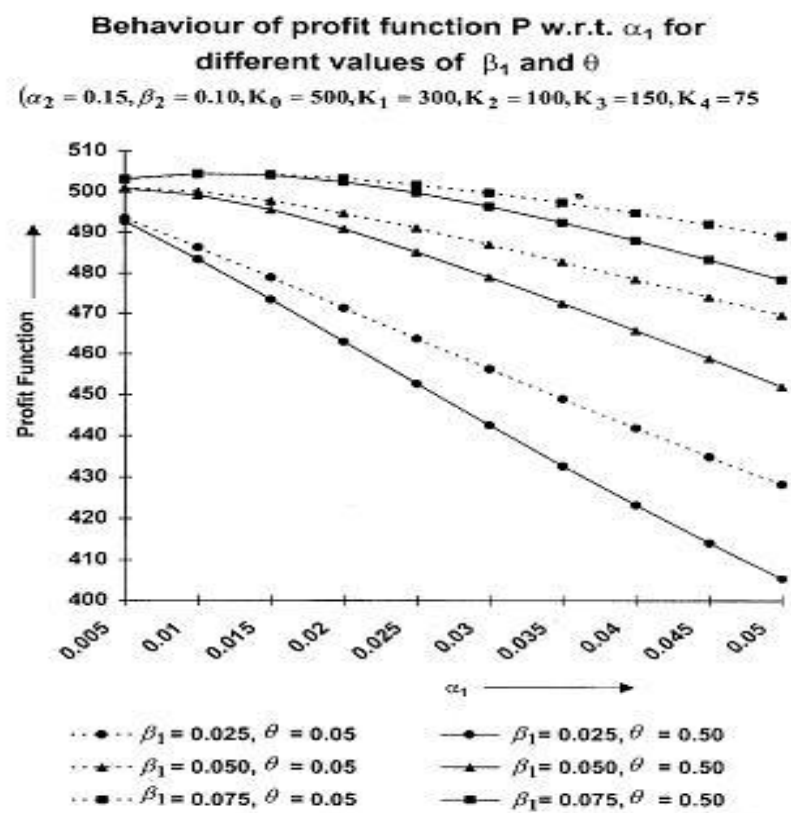

Fig 3: Behavior of Profit Function 